\title{
Review Article \\ Benign Paroxysmal Positional Vertigo of Lateral Semicircular Canal: A Systematic Review and Meta-Analysis
}

\author{
Aly M. Nagy El-Makhzangy \\ Faculty of Medicine, Ain Shams University, Cairo, Egypt \\ Correspondence should be addressed to Aly M. Nagy El-Makhzangy; Aly_El-Makhzangy@med.asu.edu.eg
}

Received 1 August 2015; Accepted 15 November 2015

Academic Editor: Jens Büntzel

Copyright ( 2015 Aly M. Nagy El-Makhzangy. This is an open access article distributed under the Creative Commons Attribution License, which permits unrestricted use, distribution, and reproduction in any medium, provided the original work is properly cited.

Benign paroxysmal positional vertigo (BPPV) is the most common cause of vertigo of peripheral origin. The lateral semicircular canal (LSCC) follows the posterior semicircular canal (PSCC) as the site of pathology in the majority of patients. Therapy, aiming at relocating particles causing aberrant LSCC stimulation has been applied by forced prolonged positioning, barbecue, and particle repositioning maneuvers. Results of the different techniques are variable. This systematic review/meta-analysis aimed to find out which therapy technique yields higher cure rates. MedLine database provided at National Library of Medicine was searched for randomized controlled trials comparing results of different therapeutic techniques for patients with LSCC BPPV. For studies included in qualitative analysis/synthesis, the following were collected independently by the author: number of participants, count of patients with geotropic and apogeotropic LSCC in each treatment group, and resolution of vertigo/nystagmus assessed by symptomatic improvement and negative supine roll test 1-24 hours following intervention considering the type of LSCC BPPV (geotropic/apogeotropic). Level Ia evidence (systematic review of RCTs) shows superiority of Gufoni maneuver over sham for both geotropic and apogeotropic LSCC BPPV. Comparisons between different therapeutic maneuvers for LSCC BPPV based on results of published RCTs could not be set.

\section{Introduction}

Benign paroxysmal positional vertigo (BPPV) is the commonest cause of peripheral vertigo [1]. Cupulolithiasis [2] and canalolithiasis [3] of the semicircular canals are the theories of the underlying mechanisms. The most commonly affected canal is the posterior $(64-88 \%)[4,5]$. The lateral semicircular canal (LSCC) follows (21-31\%) [6-8].

LSCC BPPV presents with short-lived, repeating attacks of head spinning sensation on turning the head, or whole body, while lying down, nausea, and vomiting, usually with no hearing affliction or neurological manifestations. The supine roll test (SRT) [9] confirms the diagnosis, helps to determine method of treatment, and shows its success, or otherwise. The test is performed by briskly turning the head of the patient in the supine position, approximately $90^{\circ}$ to each side. The examiner notes whether the resulting nystagmus beats towards or away from the ground (geotropic or apogeotropic, resp.) and to which side it is more intense, left or right.
In geotropic LSCC BPPV, where otoconia are in the nonampullary end, an ampullopetal stimulatory endolymph flow results on turning the involved side towards the ground [10]. In the rare apogeotropic LSCC BPPV, where otoconia are near the ampullary end or attached to the cupula (cupulolithiasis) an ampullofugal, inhibitory endolymph flow results on turning the involved side towards the ground [11]. An even rarer type resulting from presence of otoconia in both the ampullated and nonampullated parts of the LSCC causes a direction-fixed nystagmus on turning the patient's head to either side [12].

Treatment methods depend on displacing otoconia out of the LSCC by allowing them to settle down by forced prolonged positioning (FPP) [13] or agitating them out by head and body manipulations (barbecue maneuver [14], therapeutic head-shaking [13], Gufoni maneuver [15], and the modified Semont maneuver [16]).

In FPP [13], the patient is instructed to lie on the unaffected side for 12 hours. 
The barbecue maneuver [14] is performed starting with the patient in the supine position and nose up. The head is rapidly turned $90^{\circ}$ towards the unaffected ear and position maintained until the resulting nystagmus abates (in 30-60 seconds). Then the patient takes a lateral decubitus position, with the unaffected ear down, to facilitate a further speedy $90^{\circ}$ head rotation to a nose-down head position, which is also maintained for 30-60 seconds. Next, the patient lies prone and a further $90^{\circ}$ head rotation makes the affected ear down. This last position is also maintained for 30-60 seconds and then the patient sits at the side of the examination couch.

Therapeutic head-shaking [13] is performed by shaking the head sideways at a rate of about 3 times a second for 15 seconds, with the patient seated and the head pitched forward by $30^{\circ}$, to make the LSCC horizontal.

The Gufoni maneuver (GM) [15] starts with the patient sitting at the side of the examination couch and then rapidly swaying the trunk to one side (the unaffected side in geotropic LSCC BPPV or the affected side in the apogeotropic type), so that the head rests at the side of the couch. This position is maintained for 1-2 minutes. This is followed by turning the head $45^{\circ}$, with nose down, in the geotropic type, or nose up, in the apogeotropic type, and this position is maintained for 2-3 minutes. The patient then returns to the sitting position.

A modification of GM by Testa and collaborators [17] consists of a pause, midway between the sitting position and the side-lying position for 15 seconds during the lateral trunk sway, followed by slow completion of the movement until the head rests at the side of the couch. Further manipulations are similar to GM.

The modified Semont (Casani's) maneuver [16] and GM have some similarities. The differences between the 2 procedures are whether the patient's side-lying position is maintained for 1-2 minutes prior to $45^{\circ}$ head-turning, in GM $[15,16]$, or not, in the modified Semont maneuver, and the direction of head-turning: upwards in GM and downwards in modified Semont maneuver, for apogeotropic LSCC BPPV.

Success of all the different maneuvers, except for therapeutic head-shaking, for treatment of LSCC BPPV depends on correct identification of the diseased side. The side of the faster nystagmus and more intense vertigo on SRT indicates the diseased LSCC in the geotropic type, while the side of the slower, less intense nystagmus is the afflicted one in apogeotropic type. However, for the unaided eye of the examiner, and without video-nystagmography (VNG), finding out the diseased side can sometimes be difficult. To overcome this issue the "bow and lean" test has been devised depending on the fact that the LSCC subtends an angle of approximately $30^{\circ}$ with the horizontal plane [18]. More recently, $180^{\circ}$ of head rotation during the SRT, instead of $90^{\circ}$, has been found to increase the slow-phase velocity of the resultant nystagmus and thus may help to correctly identify the diseased LSCC [19].

Rationale. At least 6 different methods for treatment of LSCC BPPV are described in the literature [13-17] with cure rates varying from 74 to $94 \%$. The aim of this review/meta-analysis is to compare instantaneous results of different methods of treatment of LSCC BPPV as assessed by patient symptoms and SRT, published in randomized controlled trials, in order to help clinicians choose among them guided by the best possible available evidence. However, the choice of the method of treatment is always governed by patient specific factors, as well as training and experience of the provider of therapy.

\section{Methodology}

Electronic search of MedLine database is available at the National Library of Medicine (NLM) (http://www.ncbi.nlm .nih.gov/pubmed/) on December, 25, 2013, using the following terms: (lateral canal benign paroxysmal positional vertigo) OR (horizontal canal benign paroxysmal positional vertigo) in all fields limiting the type of studies to randomized clinical trials. No limitations on the year or language of publication of the articles were applied in the search.

Updates on new publications containing both search terms were posted by e-mail to the author through an automatic service provided by the NLM. Additional publications were sought by reviewing the references of the articles yielded by the electronic search.

Abstracts of retrieved articles were screened for relevance. Full text of relevant articles was obtained for more detailed reviewing considering participants (patients with geotropic or apogeotropic LSCC BPPV), alternative interventions/comparisons (FPP, barbecue maneuver, GM, modified Semont's maneuver, modified GM, head-shaking, or sham), outcomes (symptomatic improvement and response to SRT, 1-24 hours following intervention), and study design (double or triple armed RCT).

Risk of bias at study level (method of randomization, allocation concealment) and at outcome level (appropriateness of evaluation and analysis of results of intervention) was assessed as adequate or otherwise prior to inclusion in the quantitative analysis by reviewing the methodology section for at least one step taken to minimize the bias at the steps of participant inclusion/exclusion criteria, intervention group assignment, and outcome evaluation.

A negative response to SRT was taken as an implication that relief of symptoms occurred. Follow-up periods across studies were variable; thus only outcomes at 1-24 hours following interventions were analyzed.

Data extraction including number of patients receiving alternative interventions, method of evaluation of response to interventions, and number of respondent patients with favorable outcome(s) for performing meta-analysis using Review Manager 5 software [25] was done. Odds ratio (OR) of nonrespondent patients was the principal summary measure. The software calculates heterogeneity measures including Cochrane $Q\left(\chi^{2}\right), I^{2}$, and $\tau^{2}$, as well as generating forest plots.

Comparisons of cure rates of different methods of treatment were set by combining results from RCTs applying similar interventions, selecting results from only 2 arms of triple armed RCTs if required.

Due to the difference in resolution rates of geotropic and apogeotropic subtypes of LSCC BPPV, stratified analysis was performed to test whether the outcomes are affected by both subtype and method of therapy independently or, otherwise, prior to meta-analysis. 


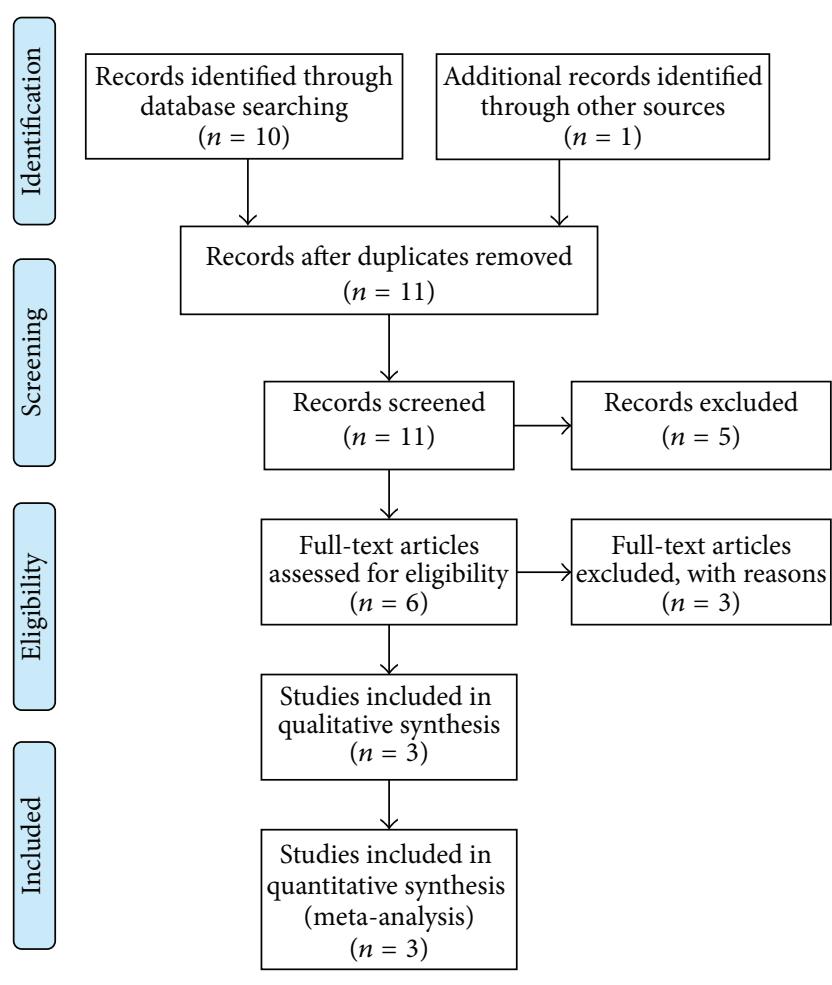

Figure 1: PRISMA flow diagram.

\section{Results}

3.1. Study Selection. Ten articles [17, 20-23, 26-30] were retrieved by the electronic search. An additional article [24] was given from back references of the articles by Kim and colleagues [21, 22]. Figure 1 shows the Preferred Reporting Items for Systematic Reviews and Meta-Analyses (PRISMA) flow diagram through which identified articles were screened and assessed for eligibility and inclusion in quantitative analysis.

3.2. Excluded Articles with Reasons for Exclusion. The article by Cambi and colleagues [26] discussed positional downbeating nystagmus which, if not due to central causes, indicates superior semicircular canal BPPV and thus was considered irrelevant.

The incidence of BPPV following the use of mallet osteotomes versus screwable osteotomes for closed sinus floor elevation was compared in a RCT [27]. PSCC BPPV occurred in $3 \%$ of patients in which the mallet osteotomes were used. This article was also considered irrelevant.

The article [28] in which only 13 patients with multiple canal BPPV, posterior and lateral, have been analyzed was also ruled out.

The article [29] that discussed the diagnostic value of different positioning tests in diagnosis of BPPV concerned patients with PSCC and only one patient had dual canal affliction, posterior and lateral, and thus was excluded.

The last of the excluded RCTs [30] due to irrelevance was reporting results of 2 different techniques for treatment of PSCC BPPV, not LSCC BPPV.
3.3. Studies Considered for Meta-Analyses. In the study by Casani and colleagues [20] for patients with geotropic LSCC BPPV, 33/54 patients in the group treated by barbecue maneuver and FPP and 50/58 patients treated by GM were symptom-free one day following the interventions $(\mathrm{OR}=$ $0.25,95 \%$ confidence interval $(\mathrm{CI})=0.10-0.63)$. A second session made $38 / 54$ patients treated by barbecue maneuver and FPP and 52/58 patients treated by GM symptom-free on the next day. One month following treatment, SRT was negative in 44/54 patients in the barbecue maneuver and FPP group compared to 54/58 patients in the GM group. At the study level, methods of intervention group size determination and patient allocation to one of the alternative interventions are not given. For the outcome, a possibility of bias exists since the authors do not state that the evaluation after intervention was performed by an examiner who was blinded to the intervention applied to the patients. Moreover, the results of this study [20] cannot be used to find out the differences in effectiveness of GM and the barbecue maneuver to treat geotropic LSCC BPPV since they are reported one day following the interventions, to allow for the 12 hours required for FPP; thus the effect of the barbecue maneuver on its own is not separable from that of the FPP.

Vertigo and nystagmus resolved in 28/53, 31/64, and $12 / 48$ patients with geotropic LSCC BPPV receiving barbecue maneuver, GM, and sham, respectively (OR for barbecue maneuver versus sham $=3.36,95 \% \mathrm{CI}=1.44-7.84$, OR for $\mathrm{GM}$ versus sham $=2.82,95 \% \mathrm{CI}=1.25-6.38$, and $\mathrm{OR}$ for GM versus barbecue maneuver $=0.84,95 \% \mathrm{CI}=0.40-1.74$ ), as evaluated 30-60 minutes following treatment by another physician blinded to the therapeutic group in the RCT by Kim and colleagues [21].

In the RCT for patients with apogeotropic LSCC BPPV [22], 31/52, 25/53, and 11/49 patients had resolution of vertigo and nystagmus by one application of GM, head-shaking, and sham procedure, respectively (OR for GM versus sham = 5.10, $95 \%$ CI $=2.14-12.17$, OR for head-shaking versus sham $=$ 3.08, 95\% CI $=1.30-7.30$, and OR for GM versus headshaking $=1.65,95 \% \mathrm{CI}=0.76-3.58)$. The second application for patients with failed response, 30-60 minutes following first application, raised the rates of resolution to 38/52, 33/53, and $17 / 49$ patients by the same respective interventions. In the follow-up 4 weeks later, 51/52, 51/53, and 48/49 patients of each group had no vertigo or nystagmus.

In the two previously summarized studies [21, 22], group size determination and patient allocation to one of the alternative interventions were detailed giving a low risk of bias at study level, as well as outcome level, due to blinding of the examiner performing evaluation after treatment to the patient's group of intervention.

Seventy-two patients with both geotropic (53) and apogeotropic (19) LSCC BPPV were randomly assigned to GM or sham maneuver [23]. Only $3 / 35$ patients in the sham maneuver group had no nystagmus 1 hour following the maneuver and only 4/35 1 day following the maneuver. In contrast, $28 / 37$ patients in the GM group were free from nystagmus after 1 hour and 31/37 patients on the following day had no nystagmus. OR for results 1 day following intervention is 40.04 in favor of GM (95\% CI $=10.28-155.94)$. Risk of bias 


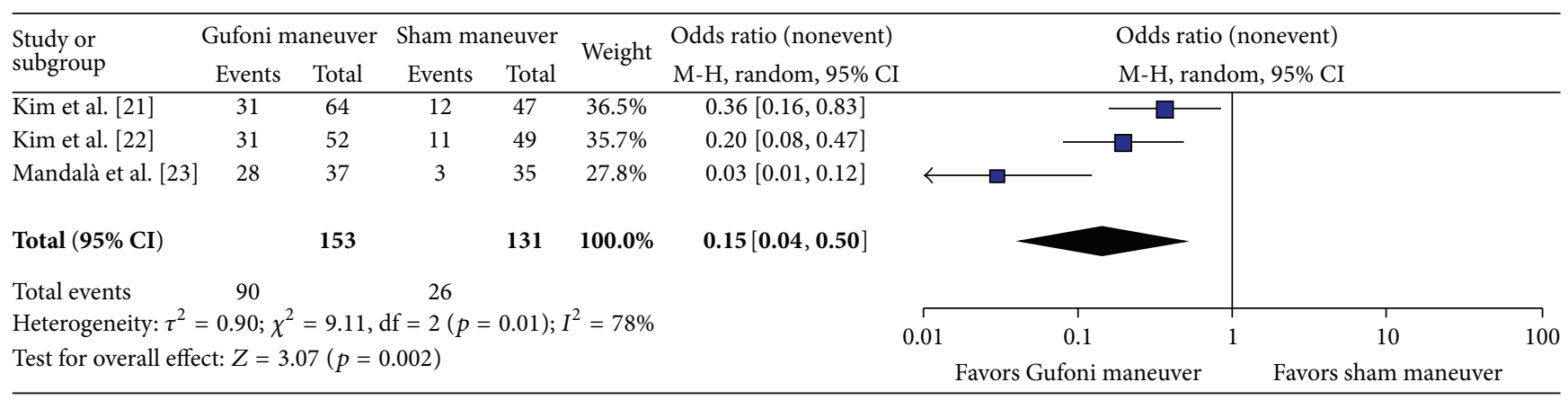

FIGURE 2: Forest plot comparison of results of 3 RCTs for resolution of vertigo and nystagmus of LSCC BPPV by Gufoni maneuver and sham maneuver as alternative interventions (generated by RevMan 5).

at study and outcome levels is low considering sample size estimation, random allocation, and evaluation of resolution by at least 2 examiners blinded to the intervention given to the patients.

Head-shaking cured apogeotropic LSCC BPPV, as evidenced by resolution of positional vertigo and nystagmus 30 minutes after the procedure in 17/51 patients in the first stage of a RCT by Oh and colleagues [24] and in 6/43 patients who were not cured by the modified Semont's maneuver, giving a total number of cures of 23/94 head-shaking maneuvers. In the same study, the modified Semont maneuver cured $7 / 52$ patients in the first stage and 7/32 patients who were not cured by head-shaking, giving a total number of cures of 14/84 modified Semont's maneuver. OR for cure is 1.62 in favor of head-shaking $(95 \% \mathrm{CI}=0.77-3.40)$. The risk of bias both at study and at outcome levels is high. No details are given to sample size determination, patient allocation was on alternate assignment basis, raising issues of periodicity, and no statement is given on whether the examiner evaluating the results of intervention was blinded or not.

A modification of GM was compared to GM in a RCT by Testa and colleagues [17]. Resolution of vertigo and nystagmus was more likely by the modified GM (40/44 in the modified GM group versus $31 / 43$ in the GM group, $\chi^{2}=5.13$, $p=0.024, \mathrm{OR}=0.26,95 \% \mathrm{CI}=0.08-0.88)$. No details on sample size determination and method of randomization are given, as well as blinding or otherwise of the examiner evaluating resolution, raising risk of bias at study and outcome levels.

Study characteristics of relevant articles reviewed for possibility of inclusion in the meta-analyses are summarized in terms of participants, interventions/comparisons, outcome(s), and study design (PICOS) in Table 1.

The effectiveness of head-shaking for treatment of apogeotropic LSCC BPPV was compared to GM [22] and to modified Semont's maneuver [24]. Though there are similarities between GM and the modified Semont maneuver, a meta-analysis comparing head-shaking to either or both cannot be set due to the differences between the 2 maneuvers. Thus, comparisons between GM and other therapeutic interventions using data published in the retrieved RCTs are not feasible.

Further results regarding the effectiveness of GM in comparison to sham procedures for treatment of geotropic/apogeotropic LSCC BPPV are given by applying meta-analysis to 3 of the included RCTs [21-23]. Results from 2 arms of each of the triple armed RCTs [21, 22] were used along with results of the RCT by Mandalà and colleagues [23]. The proportions of geotropic and apogeotropic forms in the RCTs [21-23] combined together are different from each other significantly (53 patients with geotropic LSCC BPPV and 19 patients with apogeotropic LSCC BPPV in the RCT by Mandalà and collaborators [23] versus 114 patients with geotropic LSCC BPPV and 103 patients with apogeotropic LSCC BPPV in the other 2 RCTs $[21,22], \chi^{2}=8.99, p=$ $0.0027)$. Thus, resolution of nystagmus and vertigo may be influenced not only by the interventions but also by whether the LSCC BPPV is geotropic or apogeotropic. Stratified analysis of the outcome, resolution of nystagmus, and vertigo following first application of interventions, considering the alternative maneuvers as well as the subtype of LSCC BPPV as a confounding factor, from data pooled from the 3 RCTs (Tables 2 and 3 ) showed that the null hypothesis, $H_{0}=$ There is no relationship between the two variables of interest when controlling for a third variable, can be rejected (CochranMantel-Haenszel $\chi^{2}=45.2584, p<0.0$ ). Thus, direct comparison between GM and sham procedures by performing random effect meta-analysis of the 3 RCTs [21-23] is possible (Figure 2).

\section{Discussion}

In comparison to 59 RCTs retrieved by the keyword "benign paroxysmal positional vertigo," "lateral canal benign paroxysmal positional vertigo," OR "horizontal canal benign paroxysmal positional vertigo" returned 10 RCTs by searching MedLine database, only 5 of which were found to be relevant to LSCC BPPV therapy. An increasing interest in LSCC BPPV is seen considering that 4 of the relevant RCTs [17, 21-23] were published over the past 2 years. 


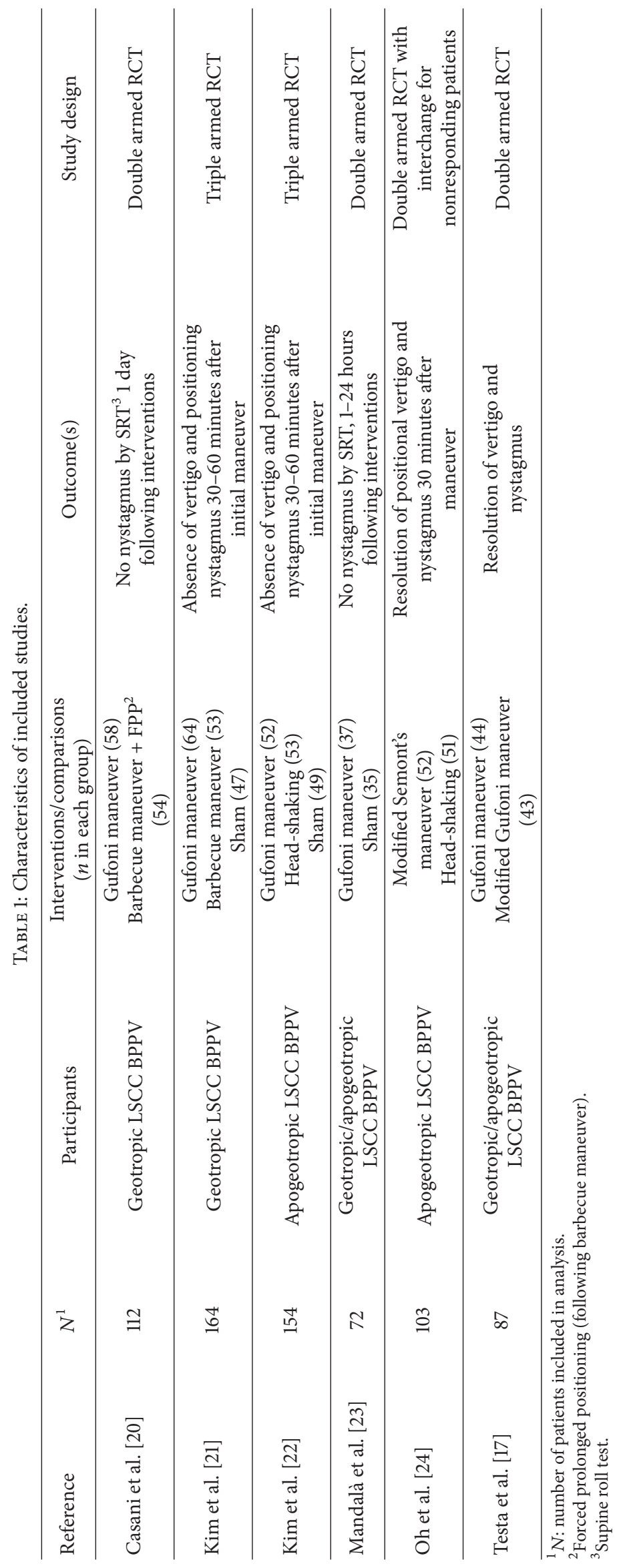


TABLE 2: Resolution of vertigo/nystagmus by GM and sham maneuver (data pooled from RCTs [21-23]).

\begin{tabular}{lccc}
\hline $\begin{array}{l}\text { Resolution of } \\
\text { vertigo/nystagmus }\end{array}$ & $\begin{array}{c}\text { Gufoni } \\
\text { maneuver }\end{array}$ & $\begin{array}{c}\text { Sham } \\
\text { maneuver }\end{array}$ & Total \\
\hline Yes & 90 & 63 & 153 \\
No & 26 & 105 & 131 \\
Total & 116 & 168 & 284 \\
\hline
\end{tabular}

$\chi^{2}=42.7739, p<0.0$

TABLE 3: Resolution of vertigo and nystagmus among patients with geotropic and ageotropic LSCC BPPV (data pooled from RCTs [2123]).

\begin{tabular}{lccc}
\hline $\begin{array}{l}\text { Resolution of } \\
\text { vertigo/nystagmus }\end{array}$ & $\begin{array}{c}\text { Geotropic } \\
\text { LSCC BPPV }\end{array}$ & $\begin{array}{c}\text { Apogeotropic } \\
\text { LSCC BPPV }\end{array}$ & Total \\
\hline Yes & 70 & 71 & 141 \\
No & 46 & 97 & 143 \\
Total & 116 & 168 & 284 \\
\hline
\end{tabular}

$\chi^{2}=8.2669, p=0.004$.

This systematic review and meta-analysis is based on results of 6 RCTs $[17,20-24], 5$ of which were those retrieved by electronic searching of MedLine database and one [24] was through back references of 2 of the 5 RCTs [21, 22].

The steps of this systematic review and meta-analysis followed, as much as possible, the Preferred Reporting Items for Systematic Reviews and Meta-Analyses (PRISMA) statement [31] (See Appendix in Supplementary Material available online at http://dx.doi.org/10.1155/2015/465095).

The main finding supported by level I evidence, metaanalysis of RCTs, is the higher effectiveness of GM for treatment of geotropic and apogeotropic LSCC BPPV in comparison to sham. The same conclusion, but limited to geotropic LSCC BPPV, was given by a previously published systematic review [32]. In this systematic review/meta-analysis, stratified analysis of the main outcome, resolution of vertigo and nystagmus, by both the type of interventions and the type of LSCC BPPV, geotropic or apogeotropic, allowed setting metaanalysis utilizing results of the 3 included RCTs [21-23] to include both types of LSCC BPPV, given that the outcome variable is shown to be significantly affected by the type of intervention, even after control for the type of LSCC BPPV as a confounder.

A significantly high level of heterogeneity among the 3 RCTs [21-23] included in the quantitative analysis is due to the lesser resolution rates in the studies by Kim and colleagues $[21,22]$ in comparison to that by Mandalà and collaborators [23] and the wide confidence interval of the OR estimate for the RCT by Mandalà et al. [23], due to the small sample size of that study.

Therefore, up to date, the clinician's choice of the therapeutic intervention for LSCC BPPV for a particular patient will be based mainly not on evidence derived from RCTs but on specific patient attributes, like the body-built, presence or otherwise of cervical and/or lumbar spine disease, and whether or not the patient can remain for 12 hours in a lateral decubitus if FPP is resorted to, which can, at least in theory, raise the risk of rethrombosis in patients with history of deep vein thrombosis (DVT).

With the occasional difficulties of identifying the side and type of LSCC BPPV taken into consideration, future research, ideally well-designed RCTs, is required to help clinicians choose among the different therapeutic techniques especially between therapeutic head-shaking, which can be effective for LSCC BPPV irrespective of the type and side of affliction, and other canalith repositioning procedures.

\section{Disclosure}

This paper was presented at the Second Asian Clinical Congress, April 3-5, 2014, Kyoto, Japan.

\section{Conflict of Interests}

The author declares that there is no conflict of interests regarding the publication of this paper.

\section{References}

[1] S.-H. Lee and J. S. Kim, "Benign paroxysmal positional vertigo," Journal of Clinical Neurology, vol. 6, no. 2, pp. 51-63, 2010.

[2] H. F. Schuknecht, "Cupulolithiasis," Archives of Otolaryngology, vol. 90, no. 6, pp. 765-778, 1969.

[3] L. S. Parnes and J. A. McClure, "Free-floating endolymph particles: a new operative finding during posterior semicircular canal occlusion," Laryngoscope, vol. 102, no. 9, pp. 988-992, 1992.

[4] F. Mosca and M. Morano, "Benign paroxysmal positional vertigo, incidence and treatment," Annales d'Oto-Laryngologie et de Chirurgie Cervico-Faciale, vol. 118, no. 2, pp. 95-101, 2001.

[5] E. Prokopakis, I. M. Vlastos, M. Tsagournisakis, P. Christodoulou, H. Kawauchi, and G. Velegrakis, "Canalith repositioning procedures among 965 patients with benign paroxysmal positional vertigo," Audiology and Neurotology, vol. 18, no. 2, pp. 8388, 2013.

[6] J. A. Lopez-Escamez, M. I. Molina, M. J. Gamiz et al., "Multiple positional nystagmus suggests multiple canal involvement in benign paroxysmal positional vertigo," Acta Oto-Laryngologica, vol. 125, no. 9, pp. 954-961, 2005.

[7] S. Y. Moon, J. S. Kim, B.-K. Kim et al., "Clinical characteristics of benign paroxysmal positional vertigo in Korea: a multicenter study," Journal of Korean Medical Science, vol. 21, no. 3, pp. 539543, 2006.

[8] Y. Chen, J.-H. Zhuang, Z.-X. Zhao, Y.-C. Li, and Z. Jin, “Clinical features of horizontal semicircular canal benign paroxysmal positional vertigo," Zhonghua Er Bi Yan Hou Tou Jing Wai Ke Za Zhi, vol. 47, no. 12, pp. 987-990, 2012 (Chinese).

[9] J. A. McClure, "Horizontal canal BPV," Journal of Otolaryngology, vol. 14, no. 1, pp. 30-35, 1985.

[10] P. Pagnini, D. Nuti, and P. Vannucchi, "Benign paroxysmal vertigo of the horizontal canal," ORL: Journal for Oto-RhinoLaryngology and Its Related Specialties, vol. 51, no. 3, pp. 161-170, 1989.

[11] P. Pagnini, P. Vannucchi, and D. Nuti, "Le nystagmus apogeotropique dans la vertige paroxystique positionelle benign du canal semicirculaire horizontal," La Revue d'Otoneurologie Française, vol. 12, pp. 304-307, 1994. 
[12] L. Califano, A. Vassallo, M. G. Melillo, S. Mazzone, and F. Salafia, "Direction-fixed paroxysmal nystagmus lateral canal benign paroxysmal positioning vertigo (BPPV): another form of lateral canalolithiasis," Acta Otorhinolaryngologica Italica, vol. 33, no. 4, pp. 254-260, 2013.

[13] P. Vannucchi, B. Giannoni, and P. Pagnini, "Treatment of lateral semicircular canal benign paroxysmal positional vertigo," Journal of Vestibular Research, vol. 7, pp. 1-6, 1997.

[14] T. Lempert and K. Tiel-Wilck, "A positional maneuver for treatment of horizontal canal benign positional vertigo," Laryngoscope, vol. 106, no. 4, pp. 476-478, 1996.

[15] M. Gufoni and L. Mastrosimone, "Trattamento con manovra di riposizionamento per la canalolitiasi orizzontale," Acta Otorhinolaryngologica Italica, vol. 18, pp. 363-367, 1998.

[16] A. P. Casani, G. Vannucci, B. Fattori, and S. Berrettini, "The treatment of horizontal canal positional vertigo: our experience in 66 cases," Laryngoscope, vol. 112, no. 1, pp. 172-178, 2002.

[17] D. Testa, G. Castaldo, C. De Santis, A. Trusio, and G. Motta, "Treatment of horizontal canal benign paroxysmal positional vertigo: a new rehabilitation technique," The Scientific World Journal, vol. 2012, Article ID 160475, 4 pages, 2012.

[18] Y.-H. Choung, Y. R. Shin, H. Kahng, K. Park, and S. J. Choi, "'Bow and lean test' to determine the affected ear of horizontal canal benign paroxysmal positional vertigo," Laryngoscope, vol. 116, no. 10, pp. 1776-1781, 2006.

[19] H. J. Lim, K. Park, H. Y. Park, and Y.-H. Choung, "The significance of 180-degree head rotation in supine roll test for horizontal canal benign paroxysmal positional vertigo," Otology \& Neurotology, vol. 34, no. 4, pp. 736-742, 2013.

[20] A. P. Casani, A. Nacci, I. Dallan, E. Panicucci, M. Gufoni, and S. Sellari-Franceschini, "Horizontal semicircular canal benign paroxysmal positional vertigo: effectiveness of two different methods of treatment," Audiology and Neurotology, vol. 16, no. 3, pp. 175-184, 2011.

[21] J. S. Kim, S.-Y. Oh, S.-H. Lee et al., "Randomized clinical trial for geotropic horizontal canal benign paroxysmal positional vertigo," Neurology, vol. 79, no. 7, pp. 700-707, 2012.

[22] J. S. Kim, S. Y. Oh, S. H. Lee et al., "Randomized clinical trial for apogeotropic horizontal canal benign paroxysmal positional vertigo," Neurology, vol. 78, no. 3, pp. 159-166, 2012.

[23] M. Mandalà, E. Pepponi, G. P. Santoro et al., "Double-blind randomized trial on the efficacy of the Gufoni maneuver for treatment of lateral canal BPPV,' Laryngoscope, vol. 123, no. 7, pp. 1782-1786, 2013.

[24] S.-Y. Oh, J.-S. Kim, S.-H. Jeong et al., “Treatment of apogeotropic benign positional vertigo: comparison of therapeutic headshaking and modified Semont maneuver," Journal of Neurology, vol. 256, no. 8, pp. 1330-1336, 2009.

[25] Review Manager (RevMan) [Computer Program]. Version 5.2, The Nordic Cochrane Centre, The Cochrane Collaboration, Copenhagen, Denmark, 2012.

[26] J. Cambi, S. Astore, M. Mandalà, F. Trabalzini, and D. Nuti, "Natural course of positional down-beating nystagmus of peripheral origin," Journal of Neurology, vol. 260, no. 6, pp. 1489-1496, 2013.

[27] G. Sammartino, M. Mariniello, and M. S. Scaravilli, "Benign paroxysmal positional vertigo following closed sinus floor elevation procedure: mallet osteotomes vs. screwable osteotomes. A triple blind randomized controlled trial," Clinical Oral Implants Research, vol. 22, no. 6, pp. 669-672, 2011.
[28] H. S. Cohen and H. Sangi-Haghpeykar, "Canalith repositioning variations for benign paroxysmal positional vertigo," Otolaryngology\&Head and Neck Surgery, vol. 143, no. 3, pp. 405$412,2010$.

[29] Y. Ou, X. Liang, Y. Zheng et al., "Value of the different positioning tests in diagnosis of benign paroxysmal positional vertigo," Lin Chuang Er Bi Yan HouKeZaZhi, vol. 19, pp. 1032-1034, 2005 (Chinese).

[30] L. Califano, P. G. Capparuccia, D. Di Maria, M. G. Melillo, and D. Villari, "Treatment of benign paroxysmal positional vertigo of posterior semicircular canal by 'Quick Liberatory Rotation Manoeuvre,' Acta Otorhinolaryngologica Italica, vol. 23, no. 3, pp. 161-167, 2003.

[31] D. Moher, A. Liberati, J. Tetzlaff, D. G. Altman, and PRISMA Group, "Preferred reporting items for systematic reviews and meta-analyses: the PRISMA statement," Annals of Internal Medicine, vol. 151, no. 4, pp. 264-269, 2009.

[32] E. M. J. M. van den Broek, H. J. van der Zaag-Loonen, and T. D. Bruintjes, "Systematic review: Efficacy of gufoni maneuver for treatment of lateral canal benign paroxysmal positional vertigo with geotropic nystagmus," Otolaryngology-Head and Neck Surgery, vol. 150, no. 6, pp. 933-938, 2014. 


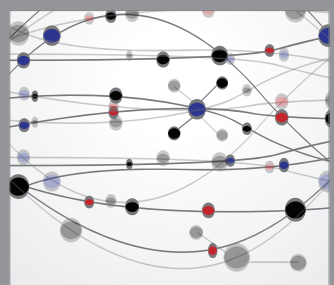

The Scientific World Journal
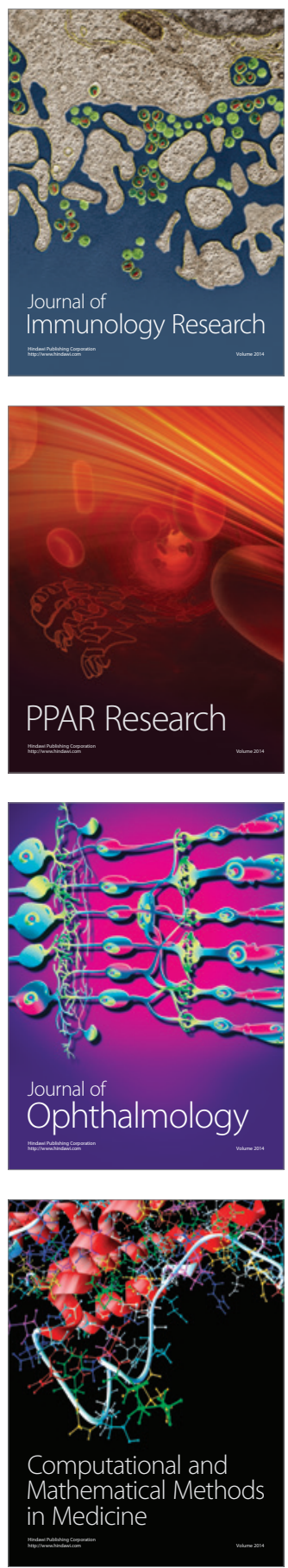

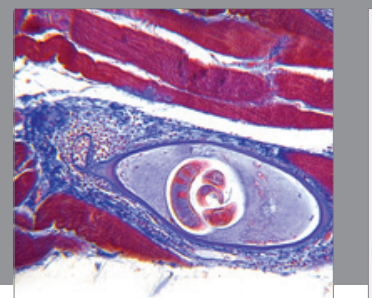

Gastroenterology

Research and Practice
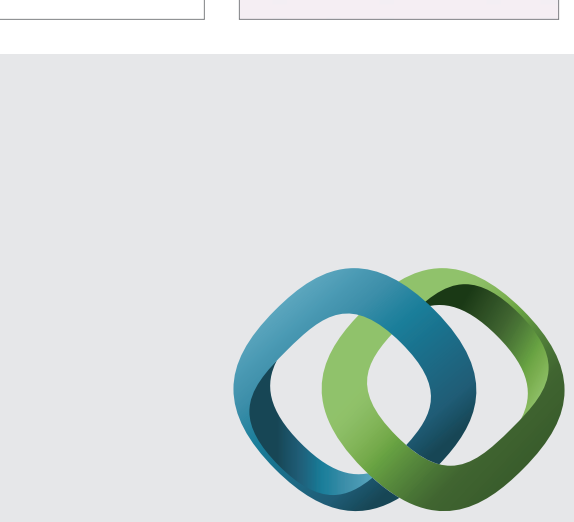

\section{Hindawi}

Submit your manuscripts at

http://www.hindawi.com
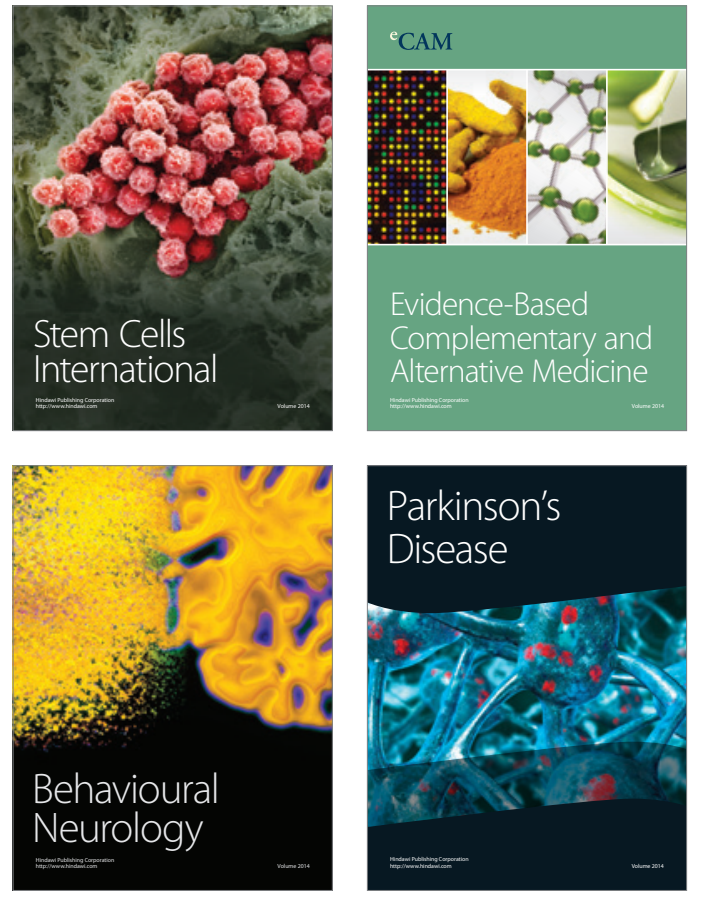
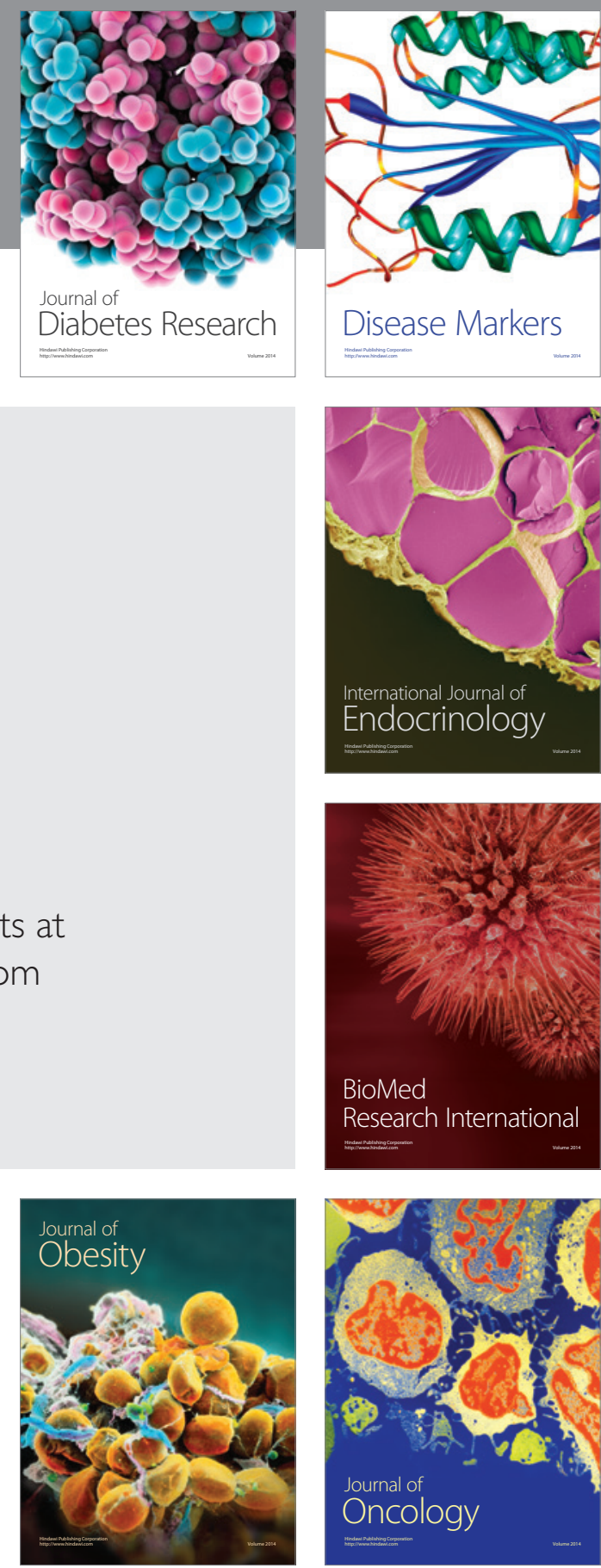

Disease Markers
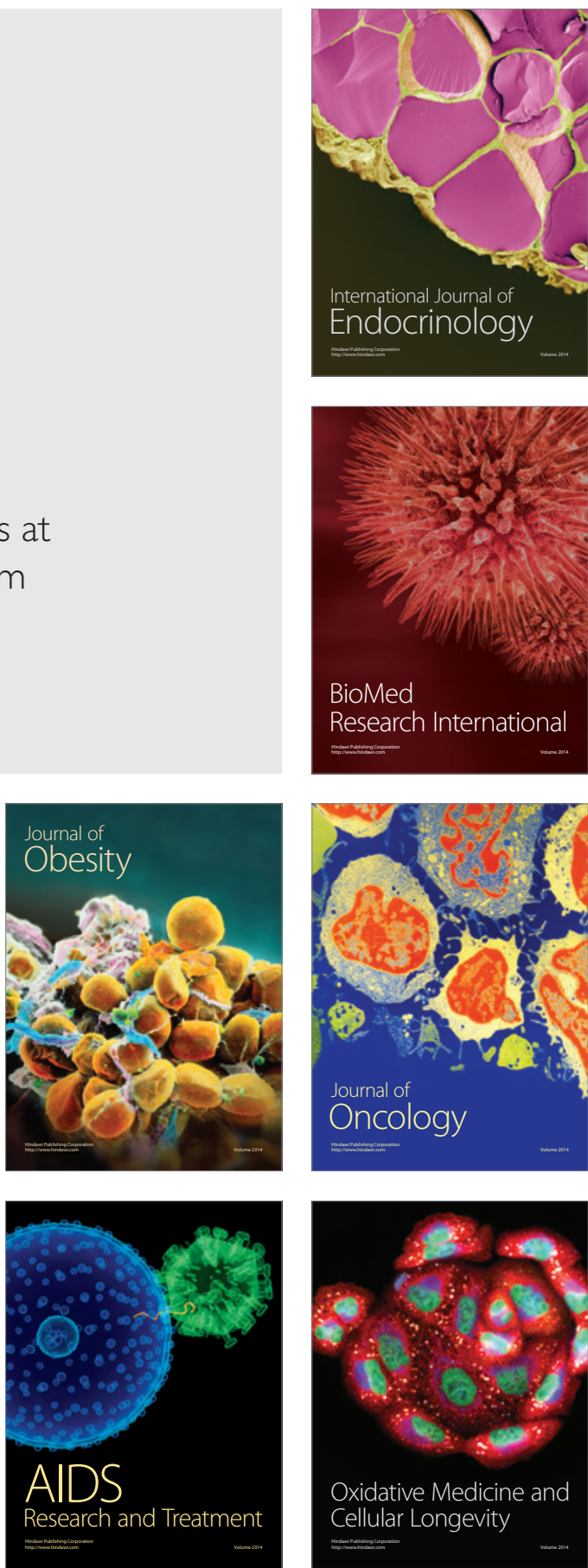